Anaesthesist 2010 - 59:5-6

DOI 10.1007/s00101-009-1649-2

Online publiziert: 23. Januar 2010

(c) Springer-Verlag 2010

\section{B. Zwissler}

Klinik für Anaesthesiologie, Klinikum der Ludwig-Maximilians-Universität München

\title{
Ein Autor und Publizist par excellence geht von Bord
}

\section{Zum Ausscheiden von Reinhard Larsen aus der Schriftleitung von Der Anaesthesist}

In diesen Tagen geht eine lange und außergewöhnlich erfolgreiche Schaffensperiode für unsere Zeitschrift zu Ende: Herr Professor Dr. Reinhard Larsen (• Abb. 1) hat sich entschlossen, nach seiner vor Kurzem erfolgten Emeritierung vom Lehrstuhl für Anästhesiologie an der Universität des Saarlandes nun auch die Koschriftleitung von Der Anaesthesist abzugeben und ab Januar 2010 in jüngere Hände zu legen.

Die Beziehung zwischen Reinhard Larsen und dem Springer-Verlag reicht bis in das Jahr 1984 - mehr als 25 Jahre - zurück. Damals erschien die 1. Auflage von Anästhesie und Intensivmedizin für die Fachpflege. Das Projekt hat sich über die Zeit zu einer Erfolgsgeschichte entwickelt, die ihresgleichen sucht. Bis heute wurden von dem Werk, das mittlerweile in der 7. Auflage erscheint, mehr als 100.00o Exemplare (mehr als 10 Mio. Seiten!) verkauft und wahrlich nicht „nur“ an Pflegekräfte in der Fachweiterbildung. Auch Generationen von Medizinstudenten haben sich damit auf ihr Staatsexamen und mancher Assistenzarzt auf seine Facharztprüfung vorbereitet. Ebenfalls 1984 erschien erstmals Anästhesie und Intensivmedizin in der Herz-, Thorax- und Gefäßchirurgie, ebenfalls ein Bestseller für den Bereich spezialisierter Fachliteratur.

Angesichts dieser außergewöhnlich erfolgreichen Zusammenarbeit auf dem Buchsektor war es nicht überraschend, dass der Springer-Verlag Mitte der 9oer Jahre die Bitte an Reinhard Larsen herantrug, sich zukünftig auch bei einem der Flaggschiffe unter den deutschsprachigen
Facharztzeitschriften, bei Der Anaesthesist zu engagieren. Dass Reinhard Larsen trotz attraktiver anderweitiger Angebote damals dieser Bitte entsprach, ist im Nachhinein als echter Glücksfall für die Zeitschrift zu werten.

Im Jahr 1995 wurde er zunächst zum Mitherausgeber für die kurz zuvor geschaffene Rubrik „Weiterbildung“ ernannt. Diese Rubrik, die seither ununterbrochen erschienen ist, wurde von ihm inhaltlich und konzeptionell wesentlich geprägt und entwickelte sich im Laufe der Jahre zu einem der Markenzeichen der Zeitschrift. Im Juli 1998 übernahm Rein- hard Larsen dann die Verantwortlichkeit für den wichtigen Bereich der Originalarbeiten, den er bis zu seinem Ausscheiden vor wenigen Wochen betreute. Dessen unbestechlicher Anspruch an wissenschaftliche Qualität und akribische redaktionelle Arbeit führten die Tradition deutschsprachiger Originalpublikationen fort, deren Grundlagen von seinen Vorgängern, z. B. Alfred Doenicke, gelegt worden waren.

Nach dem erfolgreichen Engagement in der Rubrik „Weiterbildung “ und im Bereich der Originalarbeiten erfolgte dann im August 1999 - im Zuge einer grund-

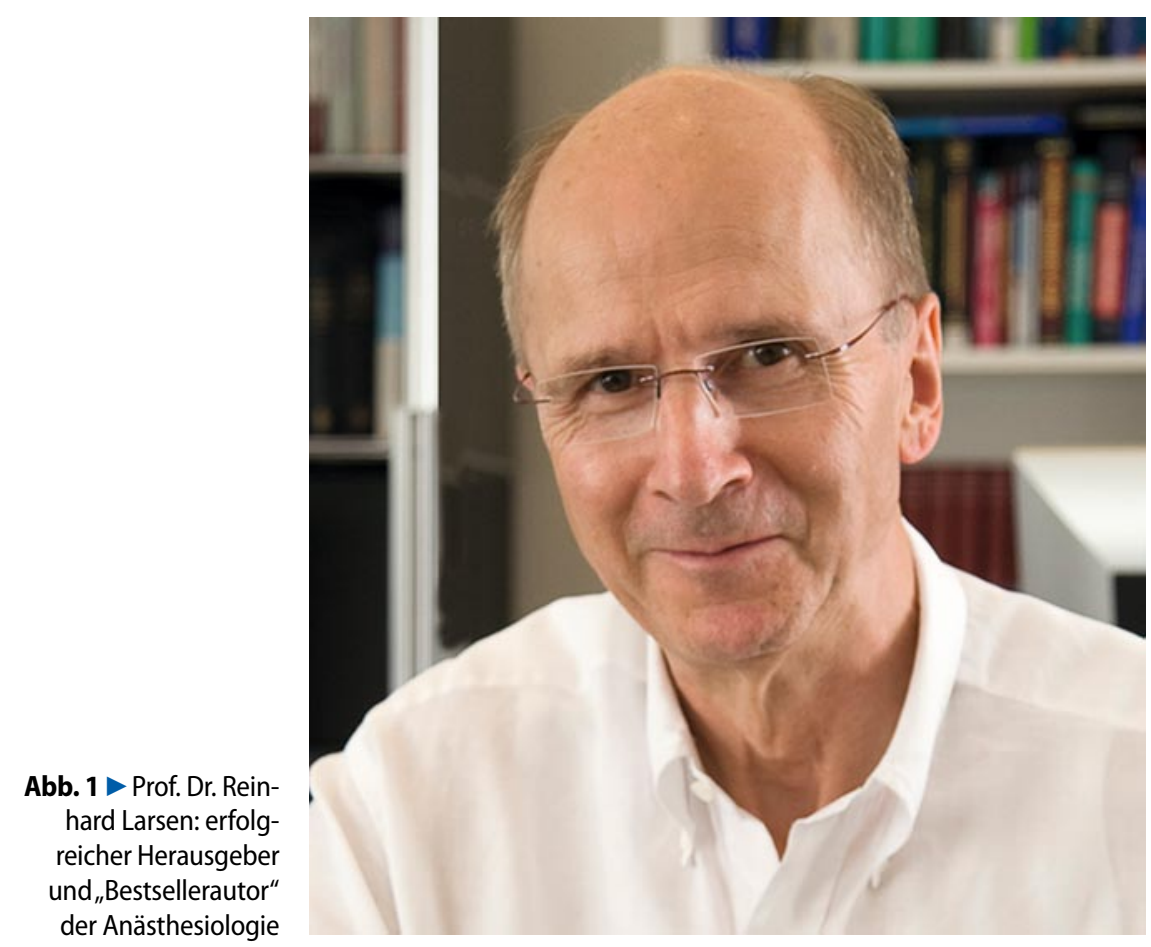


legenden Umgestaltung des Herausgeberkollegiums sowie der Etablierung neuer inhaltlicher Konzepte - die Berufung zum Schriftleiter und Mitherausgeber. Zusammen mit Prof. Dr. Dr. h.c. Klaus Peter war er seither einer von zwei Gesamtverantwortlichen für den redaktionellen Teil von Der Anaesthesist. In der Folgezeit gelang es beiden Herausgebern zusammen mit einem schlagkräftigen Team von Rubrikherausgebern und wissenschaftlichen Beiräten, Der Anaesthesist als wissenschaftlich bedeutendste Fachzeitschrift für Anästhesiologie im deutschsprachigen Raum $\mathrm{zu}$ etablieren und diese Stellung über die Jahre auszubauen.

Wer nun denkt, mit all den oben beschriebenen Aktivitäten als Autor und Publizist, Leiter einer universitären Klinik für Anästhesiologie sowie als klinisch bis zu seinem Ausscheiden im OP präsenter und aktiver Anästhesist wäre Reinhard Larsen annähernd ausgelastet gewesen, irrt jedoch gewaltig. Weitgehend zeitgleich zum Engagement in Der Anaesthesist erweiterte Reinhard Larsen seinen Wirkungskreis 1995 um die Mitherausgabe des Standardwerkes Die Intensivmedizin, das mittlerweile in der 10. Auflage erscheint. Zudem zeichnet er seit 1997 als Mitherausgeber und Autor des Praxisbuches Die Beatmung verantwortlich. Das Werk wurde nicht nur in Deutschland sehr erfolgreich verkauft; es wurde auch für den Leserkreis südlich der Alpen sprachlich adaptiert und ist dort für die italophile Leserschaft unter dem Titel $\mathrm{La}$ Respirazione artificiale, Basi e practica verfügbar. Zudem ist Reinhard Larsen auch bei anderen renommierten Verlagen ein erfolgreicher Autor.

Man kann rückblickend konstatieren, dass eine vergleichbar erfolgreiche und stetige publizistische Aktivität - als Buchautor und Herausgeber - in unserem Fachgebiet bislang ohne Beispiel ist. Die Breitenwirkung der Arbeit von Reinhard Larsen wird nicht zuletzt daran erkennbar, dass einschlägige Recherchen zum Namen „Reinhard Larsen“ in Verbindung mit „Anästhesie“ bei den gängigen Internetsuchmaschinen regelmäßig mehrere Zehntausend Einträge erbringen; damit nimmt er auch auf diesem zukunftsträchtigen Feld einen absoluten Spitzenplatz ein.
Aus all dem wird klar: Zum Jahreswechsel 2009/2010 ist ein Publizist und Autor par excellence von Bord des Anaesthesisten gegangen. Ganz persönlich bin ich Ihnen, lieber Herr Larsen, sehr dankbar, dass ich in den vergangenen zwei Jahren an Ihrer Seite in die Schriftleitung der Zeitschrift hineinwachsen und dabei von Ihrer außergewöhnlichen Erfahrung direkt profitieren durfte. Vor allem aber danke ich Ihnen stellvertretend für die gesamte Leserschaft von Der Anaesthesist, vielen Generationen von Assistenten und Fachärzten in der Anästhesiologie und Intensivmedizin, aber auch im Namen des Springer-Verlages sowie des Kollegiums der Herausgeber und wissenschaftlichen Beiräte unserer Zeitschrift für Ihr langjähriges, außergewöhnlich erfolgreiches Engagement für höchste publizistische Qualität!

Wir alle wünschen Ihnen und Ihrer Familie für die Zukunft alles erdenklich Gute und Ihnen persönlich auch im „Ruhestand" weiterhin viel Erfolg bei den schon geplanten zukünftigen Projekten.

Für Verlag und Herausgeber

Ihr

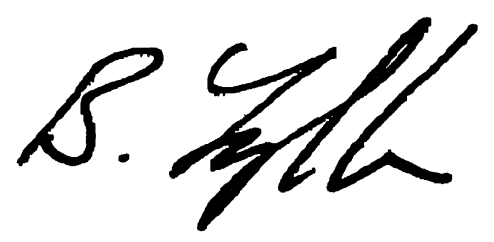

Bernhard Zwißler

\section{Korrespondenzadresse}

\section{Prof. Dr. B. Zwissler}

Klinik für Anaesthesiologie, Klinikum der Ludwig-Maximilians-Universität München Marchioninistr 15, 81377 München

Bernhard.Zwissler@med.uni-muenchen.de

\section{E. Freye \\ Opioide in der Medizin}

Heidelberg: Springer Verlag 2010, 526 S., (ISBN 978-3-540-88796-6), 79.00 EUR

Wenige Substanzen haben die Anästhesiologie, die Intensivmedizin und die Schmerztherapie derart geprägt wie die Klasse der Opioide. So basieren moderne Konzepte der balancierten oder totalintravenösen Anästhesie, der patientenkontrollierten Analgesie und der chronischen Schmerztherapie ganz überwiegend auf dem stets wachsenden Verständnis zur Schmerzentstehung und der Wirkweise dieser Substanzklasse.

Diesem Umstand wird die nunmehr in der 8. Auflage erhältliche Monographie mit dem Titel „Opioide in der Medizin“ von Enno Freye in vollem Umfang gerecht. So wird in didaktisch ausgezeichneter Weise die Pathophysiologie des Schmerzes analysiert und hieraus ein grundlegender schmerztherapeutischer Ansatz entwickelt. Als praxisorientiertes Buch mit Anspruch auf wissenschaftliche Aktualität präsentiert das Werk umfassend Indikationen und Anwendungscharakteristika der Opioide in einer modernen Schmerztherapie.

Der Autor hat Aktuelles zur Schmerzchronifizierung und zum Schmerzgedächtnis sorgfältig recherchiert und dargestellt. Er zeichnet einen vollständigen und praxisnahen Überblick zu Dosierungen, den Anwendungen der Opioide bei Neugeborenen bis hin zu geriatrischen Patienten in der Anästhesiologie, der Intensivmedizin sowie in der akuten und chronischen Schmerztherapie. Freye zeigt darüber hinaus Suchtentwicklung und komplexe Agonist- / Antagonistinteraktionen differenziert auf. Wie gewohnt, ist sowohl die sprachliche als auch graphische Aufarbeitung hervorragend gelungen. Die Abbildungen präsentieren Prinzipien von Schmerzwahrnehmung, Schmerzverarbeitung sowie pharmakologischer Interaktion mit Präzision und „aus einem Guss".

Das Buch ist sowohl als Lehrbuch als auch als Nachschlagewerk zu empfehlen und gestattet dem klinisch erfahrenen Therapeuten ebenso wie den in Weiterbildung befindlichen Medizinern und Pflegekräften, eine solide Basis und Orientierung in einem komplexen Therapiefeld zu finden.

\section{Werner, Mainz}

